\title{
Modes in silver-iodide-lined hollow metallic waveguides mapped by terahertz near-field time-domain microscopy
}

\author{
Miguel Navarro-Cia, ${ }^{1, *}$ Carlos M. Bledt, ${ }^{2}$ Miriam S. Vitiello, ${ }^{3}$ Harvey E. Beere, ${ }^{4}$ \\ David A. Ritchie, ${ }^{4}$ James A. Harrington, ${ }^{2}$ and Oleg Mitrofanov ${ }^{1}$ \\ ${ }^{1}$ Department of Electronic \& Electrical Engineering, University College London, Torrington Place, London WC1E 7JE, UK \\ ${ }^{2}$ Department of Materials Science and Engineering, Rutgers University, 607 Taylor Rd., \\ Piscataway, New Jersey 08854, USA \\ ${ }^{3}$ NEST, CNR-Istituto Nanoscienze and Scuola Normale Superiore, Piazza San Silvestro 12, Pisa 1-56127, Italy \\ ${ }^{4}$ Cavendish Laboratory, University of Cambridge, JJ Thomson Avenue, Cambridge CB3 OHE, UK \\ *Corresponding author: m.navarro-cia@ucl.ac.uk
}

Received August 6, 2012; revised October 2, 2012; accepted November 15, 2012;

posted November 16, 2012 (Doc. ID 173891); published December 12, 2012

\begin{abstract}
Thin dielectric layers inside hollow metallic waveguides are used to improve the waveguide transmission characteristics as the dominant waveguide mode changes into the hybrid $\mathrm{HE}_{11}$ mode. We investigate the effect of $1 \mu \mathrm{m}$ thick silver iodide (AgI) coatings on the fundamental modes in cylindrical waveguides at terahertz (THz) frequencies, in the regime of the dielectric layer being thinner than the optimal thickness $h_{\text {opt }}(2 \mathrm{THz}) \approx 20 \mu \mathrm{m}$. In the region of 1-3.2 THz, the lowest-order modes are similar in profile to the $\mathrm{TE}_{11}$ and $\mathrm{TM}_{11}$ modes, as determined by the timeresolved near-field measurements and verified numerically. Higher-order modes are detected experimentally as mode mixtures due to the multimode propagation. Numerical electromagnetic modeling is applied to resolve the mode structure ambiguity, allowing us to correlate experimentally detected patterns with a superposition of the $\mathrm{TM}_{11}$ and the higher-order mode, $\mathrm{TE}_{12}$. Mode profiles determined here indicate that in the regime of ultrathin dielectric $\left(h \ll 0.1 \lambda_{\text {eff }}\right)$, the dielectric layer does not transform the dominant mode into the low-loss $\mathrm{HE}_{11}$ mode. Experimental mode patterns similar to the $\mathrm{HE}_{11}$ and the $\mathrm{TE}_{01}$ modes nevertheless can be formed due to mode beating. The results indicate that the $\mathrm{Ag} / \mathrm{AgI}$ waveguides can be used for guiding $\mathrm{THz}$ waves in the $\mathrm{TE}_{01}$ mode or the $\mathrm{TE}_{12}$ mode with high discrimination against other modes. (C) 2012 Optical Society of America

OCIS codes: $\quad 110.6795,180.4243,320.7100$.
\end{abstract}

\section{INTRODUCTION}

Significant contributions to the development of terahertz (THz) dielectric-lined hollow cylindrical waveguides (also described as hollow circular waveguides) have been made by both the microwave and optical communities [1]. Experimental and theoretical development in this area started in the millimeter-wave regime [2-4]. In light of the high losses suffered by the $\mathrm{TE}_{10}$ of the traditional rectangular waveguides and the transverse electromagnetic mode of coaxial waveguides, microwave engineers shifted their interest to cylindrical waveguides and their $\mathrm{TE}_{0 n}$ modes (where $n$ is an integer and denotes radial variation) because of their low attenuation at high frequencies [5]. However, the $\mathrm{TE}_{01}$ mode is degenerate with the highly lossy $\mathrm{TM}_{11}$ mode, and researchers then examined strategies to break this degeneracy by, for instance, corrugations [6,7] or dielectric coatings [2-4, $\underline{2}, \underline{9}]$. Corrugations and dielectric inclusions were also designed to transform the $\mathrm{TE}_{11}$ eigenmode of the cylindrical waveguide to the optical-fiber-like hybrid $\mathrm{HE}_{11}$ mode. This hybrid mode is well confined in the hollow core of the waveguide, with the propagation loss and attenuation due to bends smaller than for the $\mathrm{TE}_{01}[2, \underline{3}, \underline{10,11}]$. Moreover, unlike the $\mathrm{TE}_{01}$ mode, the linearly polarized $\overline{\mathrm{HE}} \mathrm{E}_{11}$ mode facilitates the conversion to a free-space linearly polarized Gaussian beam. Corrugated waveguides have been the mainstream in high-energy science where strong interaction with an axial electron flux is pursued
$[12,13]$ and an active line in antenna research $[6,14]$. Dielectric-lined cylindrical waveguides were meanwhile developed for communication systems [2-4]; however, they were abandoned later in favor of optical fibers. These waveguides were recuperated subsequently for high-power delivery as well as for surgical applications with midinfrared lasers [15, 16]. In this regime, extensive analytical work revealed the complex development of the hybrid $\mathrm{HE}_{11}$ mode and the evolution of the other relevant modes $[\underline{10}, \underline{15}, \underline{17}, \underline{18}]$. The dielectric-lined waveguide technology for infrared waves has paved the way for reducing propagation losses in $\mathrm{THz}$ waveguides $[\underline{1}, \underline{19}]$.

Microwave, infrared, and optical regions have mature technologies as a result of extensive development. However, in the $\mathrm{THz}$ band, i.e., from 0.3 to $3 \mathrm{THz}$, components, system architectures, and experimental techniques are still emerging. In the quest to develop the $\mathrm{THz}$ band, $\mathrm{THz}$ waveguides has become an active research area [20]. The oversized corrugated and dielectric-lined cylindrical waveguides reviewed above are attractive candidates for low-loss transmission of $\mathrm{THz}$ waves. However, simply scaling corrugated microwave technology for $\mathrm{THz}$ frequencies would require extremely challenging dimensions. The fabrication difficulty for the corrugated waveguides was recently addressed by a smart arrangement of stacked rings at $0.26 \mathrm{THz}$ [21]. The dielectric-lined hollow metallic waveguides, on the other hand, can be flexible and can be fabricated at low cost [16]. In addition, in the 
mid- and far-infrared regions, they display lower losses than solid dielectric waveguides. Among the possible dielectric films available to be deposited as inner coating, silver iodide (AgI) has been extensively studied [22,23]. The reason for this choice relies on several factors: AgI is (i) nontoxic, (ii) heat resistant, (iii) highly transparent throughout the whole infrared spectrum, and (iv) compatible with Ag films. This metal is the favored material for the metallization of the hollow glass and polymer tubing. Ag has the lowest damping constant among noble metals; i.e., it is a relatively good conductor even at midinfrared. Ag toxicity is almost negligible compared to nickel, copper, or aluminum. These properties and the developed fabrication technology make the AgI-based hollow waveguides attractive for THz applications. However, increasing the thickness of the deposited dielectric is the major obstacle to extending the AgI-lined hollow metallic waveguide technology to $\mathrm{THz}$ waves, for which the optimal thickness is $10-40 \mu \mathrm{m}[24]$.

In this manuscript, we address the impact of the AgI coating on the mode structure and transmission losses for $\mathrm{THz}$ waveguides in the regime of the coating being much thinner than the wavelength. Recently the Ag waveguides with a relatively thin $1 \mu \mathrm{m} \mathrm{AgI} \mathrm{coating} \mathrm{were} \mathrm{tested} \mathrm{for} \mathrm{transmitting} \mathrm{THz} \mathrm{waves}$ [25]. The experimental results indicated the formation of the low-loss hybrid $\mathrm{HE}_{11}$ mode [25]. The thickness of the AgI coating was, however, much smaller than the estimated optimum thickness for $\mathrm{THz}$ frequencies [24]. In this regime, modal and dispersion properties of $\mathrm{Ag} / \mathrm{AgI} \mathrm{THz}$ waveguides have not been addressed thoroughly yet. Here, we combine numerical calculations with experimental $\mathrm{THz}$ near-field time-resolved mode analysis and continuous-wave (CW) mode analysis to characterize the ultrathin AgI-lined cylindrical metallic waveguides. Note that we classify dielectric films with thickness $h \ll 0.1 \lambda_{\text {eff }}$ as the ultrathin dielectrics. For comparison purposes, we also show the properties of a smooth cylindrical metallic waveguide with similar dimensions. The results obtained here also provide insight into far-field $\mathrm{CW}$ mode characterization at $\mathrm{THz}$ frequencies $[25,26,27]$.

\section{EXPERIMENTAL SETUPS AND MODELING}

The experimental time-domain setup is based on a $\mathrm{THz}$ timedomain spectroscopy (TDS) system as described in [28-31]. The waveguide sample part of the setup is shown in Fig. 1 . The incident horizontally polarized $\left(E_{x}\right)$ THz pulse generated via optical rectification is launched from the left-hand side of Fig. 1 and collimated by an Si lens ( $3 \mathrm{~mm}$ diameter). The beam is incident on a waveguide sample placed at a distance shorter than $1 \mathrm{~mm}$ from the lens. The THz pulse couples to the waveguide modes, which propagate along the waveguide and generate certain time-dependent field patterns at the output. In order to match the circularly centrosymmetric $\mathrm{THz}$ beam efficiently to the fundamental waveguide mode, $\mathrm{TE}_{11}$, and to minimize the excitation of higher-order modes, the $x y$-position of the input waveguide facet is adjusted while monitoring the output field pattern. This procedure can also be used for optimum excitation of higher-order modes, e.g., the $\mathrm{TE}_{01}$ mode [1,31]. The waveguide output is mounted on an automated translation stage and placed in close proximity to a near-field $\mathrm{THz}$ probe with an integrated subwavelength $(10 \mu \mathrm{m})$ aperture [30]. Spatial distribution of the guided

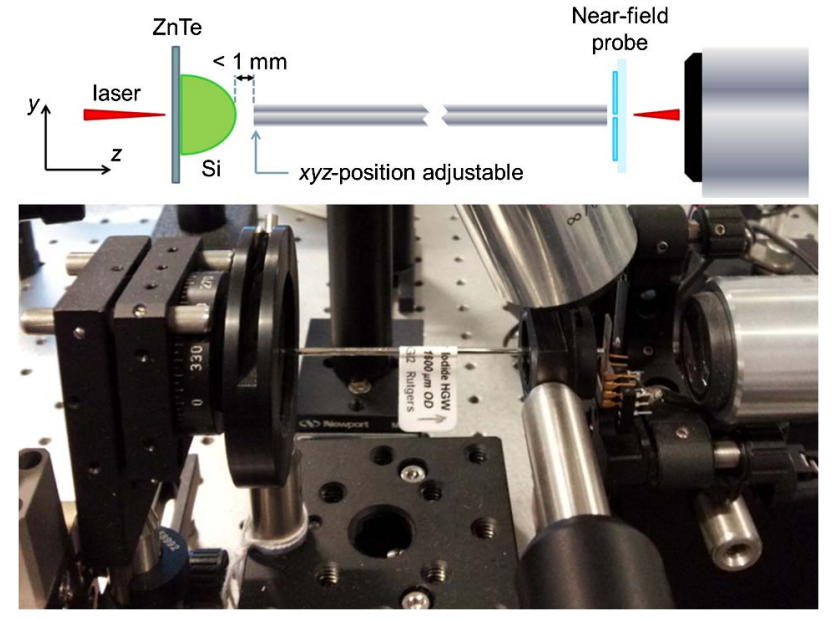

Fig. 1. (Color online) Schematic diagram (top) and picture (bottom) of the time-domain experimental setup. The waveguide can be seen in the center of the picture, whereas the subwavelength aperture near-field probe is on the right-hand side.

$\mathrm{THz}$ wave is determined by scanning the waveguide output end with respect to the probe. The change in the coupling conditions at the waveguide input during the scan is negligible because the maximum tilt of the waveguide input in this experiment is $\sim 1 \mathrm{deg}$. This system allows us to measure the output THz pulse in time (using the optical time-delay stage of the THz TDS system) at any $x y$-position of the output facet of the waveguide as described in $[1,30,31]$. It enables us to generate space-time maps, which elucidate the arrival time for waveguide modes, and two-dimensional (2D) distributions of the electric-field component $E_{x}(x, y)$ of the output THz pulse at any chosen time delay.

For CW experimental characterization, a microring distributed-feedback quantum cascade laser operating at $3.2 \mathrm{THz}$ is used as a source. The vertically emitting microring laser has a diameter $d_{R}=990 \mu \mathrm{m}$ (fabrication details are described in [27]). The device is biased, using six bonding wires along the ring. The latter procedure allows the single-mode operation and constant polarization along the whole quantum cascade laser ring. The laser is mounted on the cold finger of a liquid-helium cryostat and driven at currents of $2.04 \mathrm{~A}$ with $200 \mathrm{~ns}$ wide pulses and with an effective $0.5 \%$ duty cycle. A Picarin lens (3 cm focal length) is used to focus the azimuthally polarized $\mathrm{THz}$ beam into a $1 \mathrm{~mm}$ large pinhole kept in contact with the waveguide input. A calibrated pyroelectric detector (sensitive area about $3 \mathrm{~mm}^{2}$ ) is mounted on a $x y$ translation stage to record the far-field distribution of radiation at a distance of $\approx 2 \mathrm{~cm}$ from the waveguide output.

The waveguide in this study is a $1 \mathrm{~mm}$ inner bore diameter dielectric-lined hollow cylindrical metallic waveguide $[16,25,32]$. The AgI coating is estimated to be $1.0 \pm 0.2 \mu \mathrm{m}$ thick via the analysis of interference absorption peaks using Fourier-transform infrared spectroscopy; the waveguide sample length is $80 \pm 1 \mathrm{~mm}$ and $140 \pm 1 \mathrm{~mm}$ for the time-domain and CW experiments, respectively. To evaluate the effect of the AgI coating, a series of time-domain experiments is also performed on a similar waveguide without the AgI coating.

Before one can understand the experimental results presented here, it is helpful to review the eigenmodes supported by the hollow cylindrical metallic waveguides with and without an ultrathin dielectric coating. Although the latter 
waveguide has an exact analytical solution []ㅡ, the former does not and only approximations exist under certain conditions $[3, \underline{15}, \underline{17}, \underline{18}, 33]$. Therefore, in order to model the experiment accurately, we calculate numerically the eigenmodes and the corresponding eigenvalues (dispersion diagram) of both waveguides with the $2 \mathrm{D}$ eigenmode solver of CST Microwave Studio ${ }^{\mathrm{TM}}$.

A nonuniform tetrahedral mesh is used rather than a hexahedral grid to map accurately the curvature and the dielectric region of the cylindrical waveguide without heavy demand on computational resources. The maximum and minimum edge lengths of the tetrahedral mesh for the uncoated waveguide are 45.5 and $6.4 \mu \mathrm{m}$, respectively. For the AgI-lined waveguide, a mesh refinement has been employed on the AgI coating leading to maximum and minimum edge lengths of 38.5 and $0.4 \mu \mathrm{m}$, respectively. The metal, silver, is modeled with a conductivity $\sigma_{\mathrm{Ag}}=6.3012 \times 10^{7} \mathrm{~S} / \mathrm{m}$, whereas the AgI is considered to have a relative permittivity $\varepsilon_{r}^{\prime}=2.3$ and loss tangent $\operatorname{tg} \delta=\varepsilon_{r}^{\prime \prime} / \varepsilon_{r}^{\prime}=0.001$. To the best of our knowledge, AgI has not been characterized at the frequencies of interest of this work, and thus, the chosen loss tangent is a conjecture founded on the typical values for dielectric films at $\mathrm{THz}$ frequencies. Needless to say, the full-wave simulation of the whole setup or even the $80 \mathrm{~mm}$ long waveguide alone would lead to inaccessible computation effort.

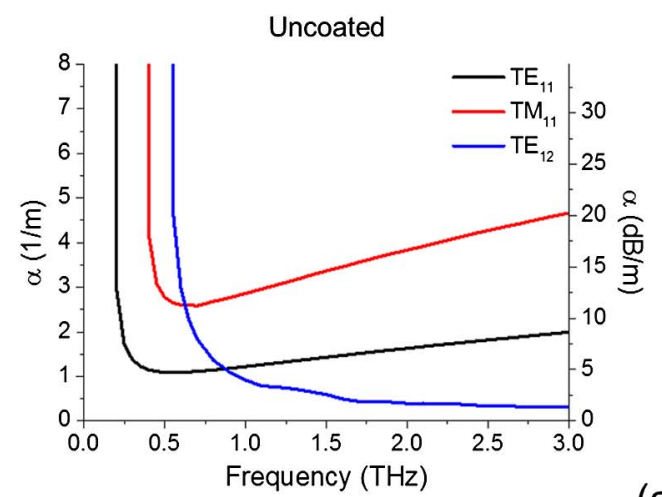

(a)
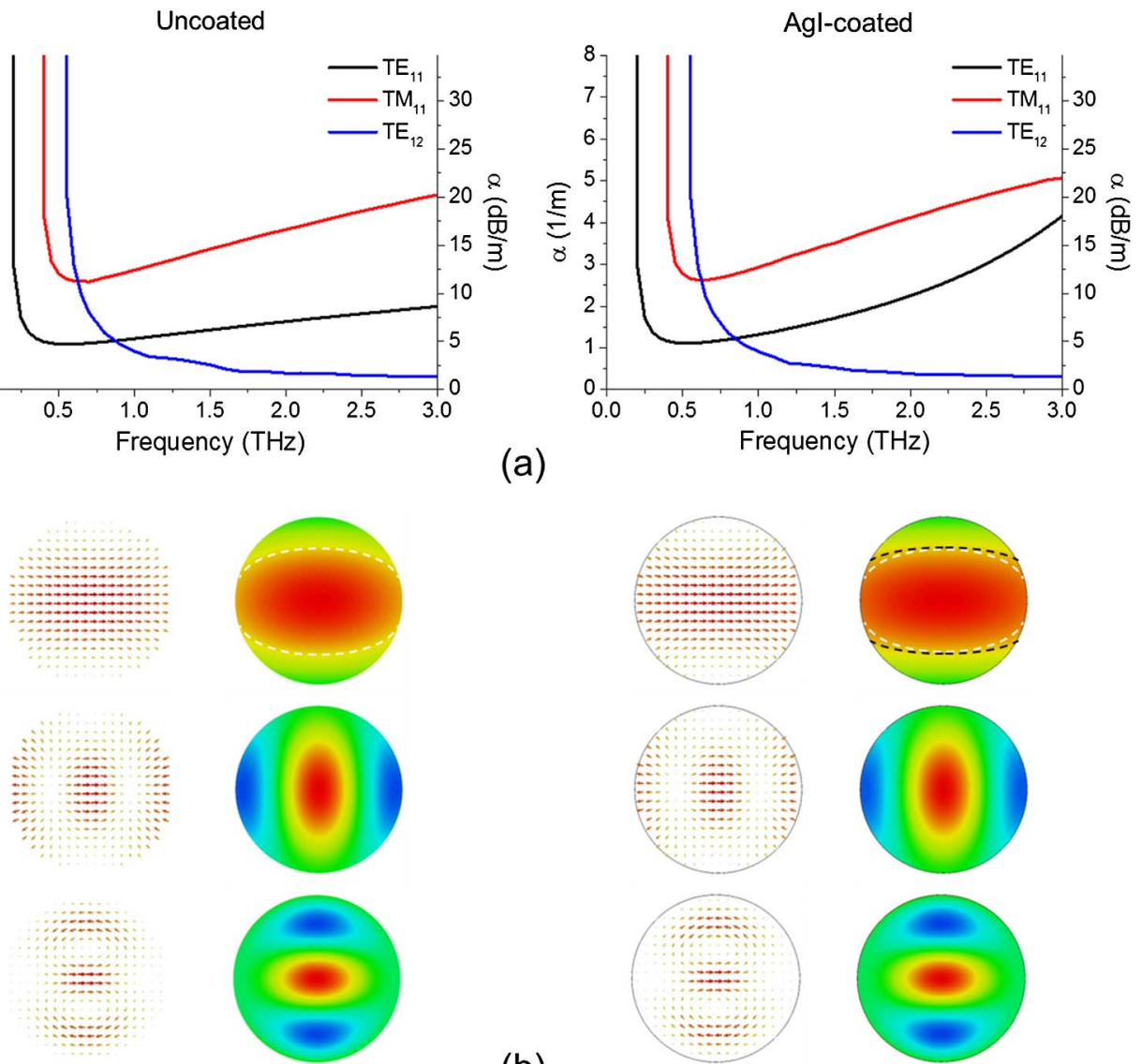

(b)

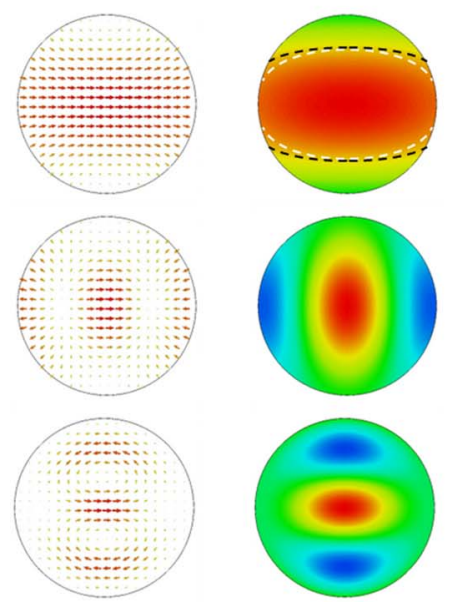

Fig. 2. (Color online) Numerical results for the uncoated (left) and AgI-coated (right) hollow cylindrical metallic waveguide (1 mm diameter). (a) Attenuation coefficient of the modes observed in the experiments: $\mathrm{TE}_{11}$ (black curve), $\mathrm{TM}_{11}$ (red curve), and $\mathrm{TE}_{12}$ (blue curve) from the lowest to the highest cutoff frequency, respectively. (b) Electric-field lines $E(x, y)$ and $E_{x}(x, y)$ color map of the aforementioned modes at 2 THz. From top to bottom: $\mathrm{TE}_{11}, \mathrm{TM}_{11}$, and $\mathrm{TE}_{12}$. The circle in the field-vector diagram represents the dielectric coating. The white and the black dashed curves show the contour of equal intensity for the uncoated and AgI-coated waveguide, respectively. To underline the change in ellipticity of the $\mathrm{TE}_{11}$ mode, the contour line for the uncoated is also included on the color map for the AgI-coated waveguide.
Figure 2 shows the attenuation constant for the $\mathrm{TE}_{11}, \mathrm{TM}_{11}$, and $\mathrm{TE}_{12}$ modes of the smooth (metal only) waveguide and for similar modes in the AgI-lined waveguide. Notice that the first 10 modes from the lowest upward are $\mathrm{TE}_{11}, \mathrm{TM}_{01}, \mathrm{TE}_{21}, \mathrm{TM}_{11}$, $\mathrm{TE}_{01}, \mathrm{TE}_{31}, \mathrm{TM}_{21}, \mathrm{TE}_{41}, \mathrm{TE}_{12}$, and $\mathrm{TM}_{02}$ [34]. However, we restrict the numerical analysis to the aforementioned three modes since they are the most likely excited modes under the on-center symmetrical illumination in our experimental system [20].

The $1 \overline{\mu m}$ thick coating has a significant impact on the atthe $\mathrm{TE}_{11}$ mode and the $\mathrm{TM}_{11}$ mode, which have a significant raction of the mode energy distributed near the waveguide [Fig. 2(b)]. For instance, at $2 \mathrm{THz}$ [the central frequency of the THz pulses in our experiment; see Fig. 3(a)], the at tenuation coefficient for the $\mathrm{TE}_{11}$ mode increases from 7.08 to $9.75 \mathrm{~dB} / \mathrm{m}$ when the AgI coating is introduced [35]. This increase can be understood qualitatively by inspecting e electric-field distribution $E_{x}(x, y)$, which shows that the strength of the field on the waveguide walls is larger in the Fig 2(b)] The coated with a thin dielectric [top row of 16.63 to $17.86 \mathrm{~dB} / \mathrm{m}$. This mode, however, remains almost unaltered when the AgI coating is introduced. The attenuation of the $\mathrm{TM}_{11}$ can arguably be expressed as the sum of the tenuation coefficient of the modes [Fig. 2(a)], especially for 


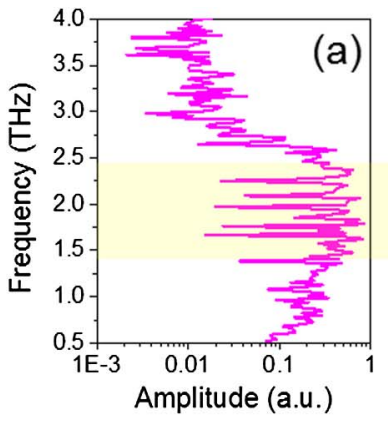

(b)
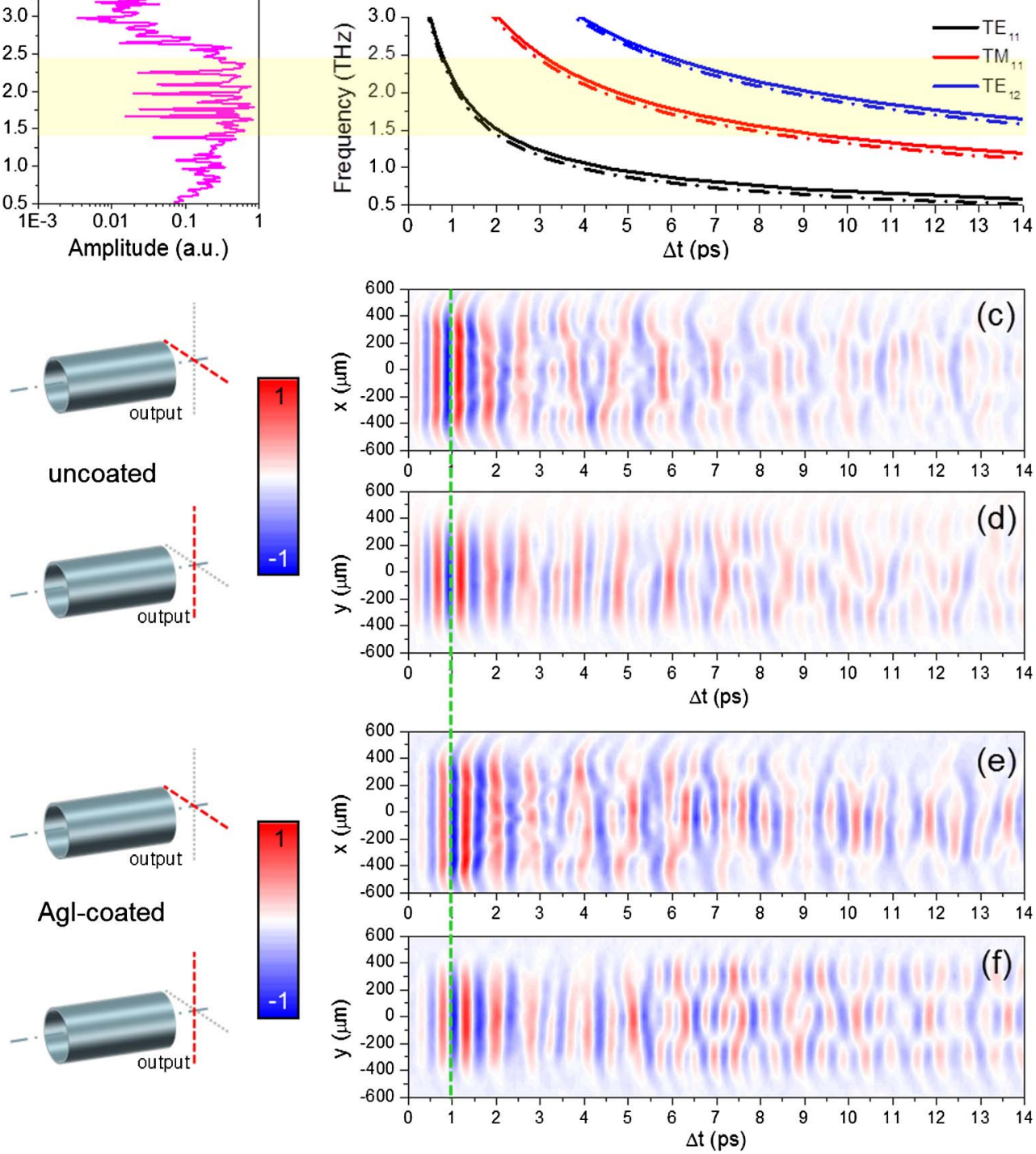

Fig. 3. (Color online) (a) THz TDS spectra measured at the output of the AgI-coated waveguide (top left). The narrow absorption lines are due to the water vapor inside the waveguides. (b) Numerically computed modal group time delays for the uncoated, dashed-dot curves, and AgI-coated waveguide, solid curves, (top right). The yellow region, i.e., $1.5-2.5 \mathrm{THz}$, highlights the frequency range with maximum energy. Schematic diagrams of the experimental linear scans and the corresponding $x t$ - and $y t$-maps for the uncoated (c), (d) and AgI-coated (e), (f) waveguide samples. The vertical green dashed line crossing panels (c)-(f) at $\Delta t=1 \mathrm{ps}$ is shown for reference.

attenuation constant of the mode in a metallic waveguide and additional loss due to absorption in the AgI-coating [middle row of Fig. 2(b)] [24]. The $\mathrm{TE}_{12}$, on the contrary, does not suffer any significant increment of the attenuation constant. This mode does not see the influence of the dielectric as a result of its confinement to the center of the waveguide. These heuristic arguments are analytically founded $[\underline{15}, \underline{17}, \underline{18}, \underline{24}]$.

Dispersion characteristics for the $\mathrm{TE}_{11}, \mathrm{TM}_{11}$, and $\mathrm{TE}_{12}$ modes in both waveguides are displayed in Fig. 3(b) in the form of modal group time delay, $\Delta t_{m}$. This delay is computed as $\Delta t_{m}=l / v_{g}$, where $l$ is the length of the waveguide and $v_{g}$ is the group velocity. The numerical results show that the $1 \mu \mathrm{m}$ thick coating has, however, a small effect on the dispersion characteristic of these modes at $\mathrm{THz}$ frequencies. Indeed, this is also confirmed by inspection of the electric-field lines
$E(x, y)$ and the electric-field component $E_{x}(x, y)$; see Fig. 2(b). From these electric-field distributions, one can detect that the fundamental mode in the AgI-coated waveguide is not as oval as in the uncoated waveguide [top row of Fig. 2(b)], whereas the other two modes remain without any appreciable alteration [middle and bottom rows of Fig. 2(b)]. For thinner dielectrics, the modal and dispersion properties of the AgI-lined cylindrical waveguide approach those of the smooth metallic cylindrical waveguide $[\underline{15}, 17,18]$. For thicker layers, the normal modes are modified to a larger extent. In particular, for the dielectric layer of few tens of micrometers, the $\mathrm{HE}_{11}$ mode forms within our frequency range [24,30,31].

After this initial numerical analysis we expect the $\mathrm{TE}_{11}$, $\mathrm{TM}_{11}$, and $\mathrm{TE}_{12}$ modes to appear within the first $14 \mathrm{ps}$ (after the arrival of the $\mathrm{THz}$ pulse in free space) for the $80 \mathrm{~mm}$ 
waveguide. Therefore, the experimental data displayed next will be shown in the time-delay span from 0 to $14 \mathrm{ps}$.

\section{EXPERIMENTAL RESULTS AND DISCUSSION}

\section{A. Space-Time Mapping of Waveguide Modes}

As we have shown previously $[1,30,31]$, the time-resolved near-field mode imaging system allows us to unveil experimentally the waveguide modal characteristics. In this section we discuss the experimental characteristics of the dielectriclined waveguides in the regime of ultrathin dielectric, for which the existing analytical approximation breaks down. The time-dependent $x$-component of the electric field, $E_{x}$, along the horizontal $(x-)$ and vertical $(y$-) axes is displayed in Figs. 3(c) and 3(e) and in Figs. 3(d) and 3(f), respectively, for the two test waveguides. Note that $\Delta t$ corresponds to the time delay with respect to free-space propagation. $\Delta \mathrm{t}$ was calibrated in the experiment for the metal-only waveguide. For the metallic waveguide, the experimental delay is matched to the delay experienced by the $\mathrm{TE}_{11}$ mode at the frequency of $2 \mathrm{THz}$ after propagation through the $80 \mathrm{~mm}$ long waveguide.

The regions of intense waves in the maps displayed in Fig. 3 reveal the propagation of different modes. The prominent pulse at the delay $\Delta t=0.5-1.5 \mathrm{ps}$ unquestionably corresponds to the fundamental mode $\mathrm{TE}_{11}$, which has the fastest group velocity among all eigenmodes. Notice that this mode tends to vanish after 3 ps because the spectral components associated with later delay times fall below the $1.5-2.5 \mathrm{THz}$ bandwidth [see Fig. 3(b), yellow shading]. We can equally well link the early arriving mode to the $\mathrm{TE}_{11}$ based on the spatial profile. According to the eigenmode analysis [Fig. 2(b)], the $E_{x}$ of the $\mathrm{TE}_{11}$ expands uniformly along the whole horizontal axis, whereas it drops well before reaching the top and bottom parts of the waveguide. This unique profile is clearly recovered by our experimental space-time maps of Figs. 3(c) and $3(\mathrm{~d})$. It is not surprising that the $\mathrm{TE}_{11}$ carries most of the energy since a circularly centrosymmetric beam has the highest correlation with the $\mathrm{TE}_{11}[\underline{12}-14, \underline{20}]$.

A $\mathrm{TE}_{11}$-like mode is also present in the $\mathrm{Ag} / \mathrm{AgI}$ waveguide. The visible delay in the dielectric-lined waveguide compared to the metal-only waveguide reflects slightly different group velocities for the dominant modes in the two waveguides. In accordance with the theory of dielectric-lined or corrugated waveguides [2-ㅜㄴ, $\underline{8}, \underline{15}-\underline{18}]$, the coating, even as thin as in our case, slows down the mode propagation. This is evident by comparing the 2D color plots of Figs. 3(c)-3(f), and it is emphasized in the top panel of Fig. 3(b), where the numerically computed modal group time delays for the uncoated and coated waveguide are displayed together. Experimentally, the $\mathrm{TE}_{11}$-like mode in the coated waveguide sample arrives around $0.13 \mathrm{ps}$ after the $\mathrm{TE}_{11}$ mode in the metal-only sample, whereas the modeling predicted 0.08 ps. The slight disagreement can be arguably assigned to the deviation of the estimated dielectric thickness and the modeled relative dielectric permittivity from the real values, pulse reshaping, and experimental uncertainties, such as roughness of the dielectric, which is known to affect the propagation of the modes [16].

It is worth mentioning that we have measured consistently around $50 \%$ lower amplitude of the $\mathrm{TE}_{11}$ for the AgI-coated waveguide compared to the uncoated one (after the optimization of the input conditions for each case). This is in qualitative accordance with the numerically computed attenuation coefficient analysis of Fig. 2(a): the $\mathrm{TE}_{11}$ experiences higher attenuation for the AgI-coated waveguide. Although the higher attenuation can be detected in our experiment, we note that quantitative evaluation of the loss in this waveguide requires longer waveguide samples $(\sim 100 \mathrm{~cm})$ in to order achieve a sufficient accuracy of $\sim \pm 0.5 \mathrm{~dB} / \mathrm{m}$.

Other modes arrive after the $\mathrm{TE}_{11}$ mode. Around $\Delta t=4 \mathrm{ps}$, one can identify the distinctive spatial profile of the $\mathrm{TM}_{11}$ mode along horizontal and vertical axes. In the $x t$ map, the electric field in the center $E_{x}(x \sim 0, y=0)$ and near the edges $E_{x}(x \sim \pm 400 \mu \mathrm{m}, y=0)$ is out of phase, whereas in the $y t$ map the electric-field component $E_{x}(x=0, y)$ reaches a maximum around $y=0$ and decays toward the edges of the waveguides. This distribution along the principal axes is in agreement with Fig. 2(b). The color pattern beyond $\Delta t=6 \mathrm{ps}$ could be traced to the $\mathrm{TE}_{12}$-mode pattern displayed in Fig. 2(b). The reader is referred to Figs. 3(e) and 3(f) for analysis of this mode because it has a higher relative amplitude in the AgI-coated waveguide. In the $y t$-map [Fig. $3(\mathrm{f})$ ], the wavefront displays phase changes near $y= \pm 200 \mu \mathrm{m}$ in the region of $\Delta t=6-14 \mathrm{ps}$. Meanwhile, the strength of the field reaches a maximum at the waveguide center and diminishes continuously at the walls in the $x$-map [Fig. 3(e)]. Such a profile is consistent with the $\mathrm{TE}_{12}$ mode [Fig. $2 \overline{(\mathrm{b})}$ ]. The $\mathrm{TE}_{12}$ mode is also more confined along $x$ than the $\mathrm{TM}_{11}$ mode along the $y$-axis and the $\mathrm{TE}_{11}$ mode along any of the principal axes.

According to the numerically computed modal time delay for frequencies 1.5-2.5 THz, we expect a mixture of the $\mathrm{TM}_{11}$ and $\mathrm{TE}_{12}$ modes (of different frequencies) within 6-10 ps. Indeed, this can be envisaged in the space-time maps by comparing comprehensively the time spans from 6 to $10 \mathrm{ps}$ and from 10 to $14 \mathrm{ps}$. The former time span has a less uniform wave pattern due to the beatings of the two modes. In the next section we will confirm the beating of the $\mathrm{TM}_{11}$ and $\mathrm{TE}_{12}$ mode within the time span $6-10$ ps by examining the wave spatial profiles (xy-maps).

As a final remark of this section, we note that the relative magnitude of the $\mathrm{TE}_{12}$ mode (and the admixture $\mathrm{TM}_{11}+\mathrm{TE}_{12}$ ) with respect to the $\mathrm{TE}_{11}$ mode is larger for the AgI-coated waveguide than for the uncoated waveguide. This is a direct consequence of the smaller effect that the dielectric coating has on the $\mathrm{TE}_{12}$ mode losses compared to the effect on the $\mathrm{TE}_{11}$ mode losses [Fig. 2(a)]. The ultrathin AgI-coated waveguide could be then exploited advantageously for $\mathrm{TE}_{12}$ mode propagation if it could be excited selectively. To this end, spatial light modulators or off-center illumination, as we have already shown for selective $\mathrm{TE}_{01}$-mode excitation (see Fig. 1 of [31]), can be used.

\section{B. Near-Field and Far-Field Waveguide Mode Imaging} The identification of modes with the help of $x t$ - and $y t$-maps is effective when the modal structure is known. However, this analysis does not give clear conclusions in complex situations of multimode propagation and for waveguides with an unknown mode structure. In such cases, images of spatial distribution of the electric-field component $E_{x}(x, y)$ are required. Even with the spatially and temporally resolved waveforms, we will see in this section that numerical analysis is required 
to achieve a full interpretation of the experimental results for the higher-order modes.

Figure $\underline{4}$ displays the 2D distribution of the electric-field component $E_{x}(x, y)$ for different time delays. At $\sim 1.5 \mathrm{ps}$ the field patterns for both waveguides account for the dominant $\mathrm{TE}_{11}$ mode [similar to the computed modes in Fig. 2(b)]. The profiles at $\sim 4.8$ ps resemble the $\mathrm{TM}_{11}$ mode with the side lobes out of phase with respect to the center spot. These two time delays correspond to the single-mode scenarios, and hence, the straightforward correlation between numerical and experimental results. However, at $\sim 7.5 \mathrm{ps}$, the field patterns for both waveguides show a concentric disk + ring configuration, which is different from the calculated normal modes (Fig. 2). The field distribution in the AgI-coated waveguide is reminiscent of the $\mathrm{TE}_{12}$, but the field distribution for the uncoated waveguide is certainly different from any of the modes supported by the waveguide.

Now the usefulness of the numerical work becomes evident. First of all, the time-resolved near-field mode map cannot be linked to any numerically computed mode. Second, the modal time delays for the given spectrum suggest the presence of several modes for the time delays larger than $4 \mathrm{ps}$. Therefore, it is reasonable to explain the observed patterns as a superposition of at least two modes. The modal dispersion results [Fig. 3(b)] restrict the attention to a limited number of modes. At $7.5 \mathrm{ps}$ our attention should be put to the $\mathrm{TE}_{12}, \mathrm{TM}_{11}, \mathrm{TE}_{01}, \mathrm{TE}_{31}, \mathrm{TM}_{21}, \mathrm{TE}_{12}$, and $\mathrm{TM}_{02}$ modes. However, we can limit the selection of modes further because the experimental setup favors coupling into the $\mathrm{TM}_{11}$ and $\mathrm{TE}_{12}$ modes [20]. Therefore, we can expect the experimental patterns in Fig. $\underline{4}$ as the admixture: $a \cdot E_{x}^{\mathrm{TM}_{11}}+b \cdot E_{x}^{\mathrm{TE}_{12}}$. The combinations of $a=-0.07$ and $b=0.9975$ for the uncoated waveguide and $a=0.05$ and $b=0.9987$ for the AgI-coated waveguide match perfectly the experimental time-resolved near-field maps in Fig. 4(c). Note that the relative amplitude coefficients $a$ and $b$ are found by a fitting procedure and are scaled to satisfy $a^{2}+b^{2}=1$ and the minus sign accounts for the $180^{\circ}$ phase shift. We emphasize that $a$ and $b$ can be interpreted as the relative mode amplitudes only for the purpose of mode analysis at a selected time delay $\Delta t$. Values of these coefficients vary with the time delay because each mode is described by a unique temporal envelope. Consequently, the different weights of the admixture for the uncoated and coated waveguides should not be attributed solely to losses due to the AgI coating [3]. The coefficients also depend on the relative group and phase delays between the modes.

We can also infer that the $\mathrm{TM}_{11}$ and $\mathrm{TE}_{12}$ modes should be present in the admixture by simple inspection of the color maps in Fig. 4(c). The images show an antinode in the waveguide center. Only the $\mathrm{TM}_{11}$ and $\mathrm{TE}_{12}$ modes (among the modes that are present at $\Delta t=7.5 \mathrm{ps}$ ) exhibit the antinode. Therefore the admixture should have at least one of these modes. The other clue comes from the in-phase ring. In order to convert side lobes of the $\mathrm{TM}_{11}$ mode into a ring, a mode pattern with the in-phase top and bottom lobes is required. The only one in the list is the $\mathrm{TE}_{12}$ mode. Likewise, in order to close an outer ring using the $\mathrm{TE}_{12}$ mode, a mode pattern with in-phase lateral lobes, such as the $\mathrm{TM}_{11}$ pattern or the $\mathrm{TE}_{31}$ pattern, is needed. We can rule out the $\mathrm{TE}_{31}$ mode because its superposition with the $\mathrm{TE}_{12}$ mode forms a ring with four nodes and four antinodes. Hence Fig. 4(c) displays a superposition of the $\mathrm{TM}_{11}$ and $\mathrm{TE}_{12}$ modes.
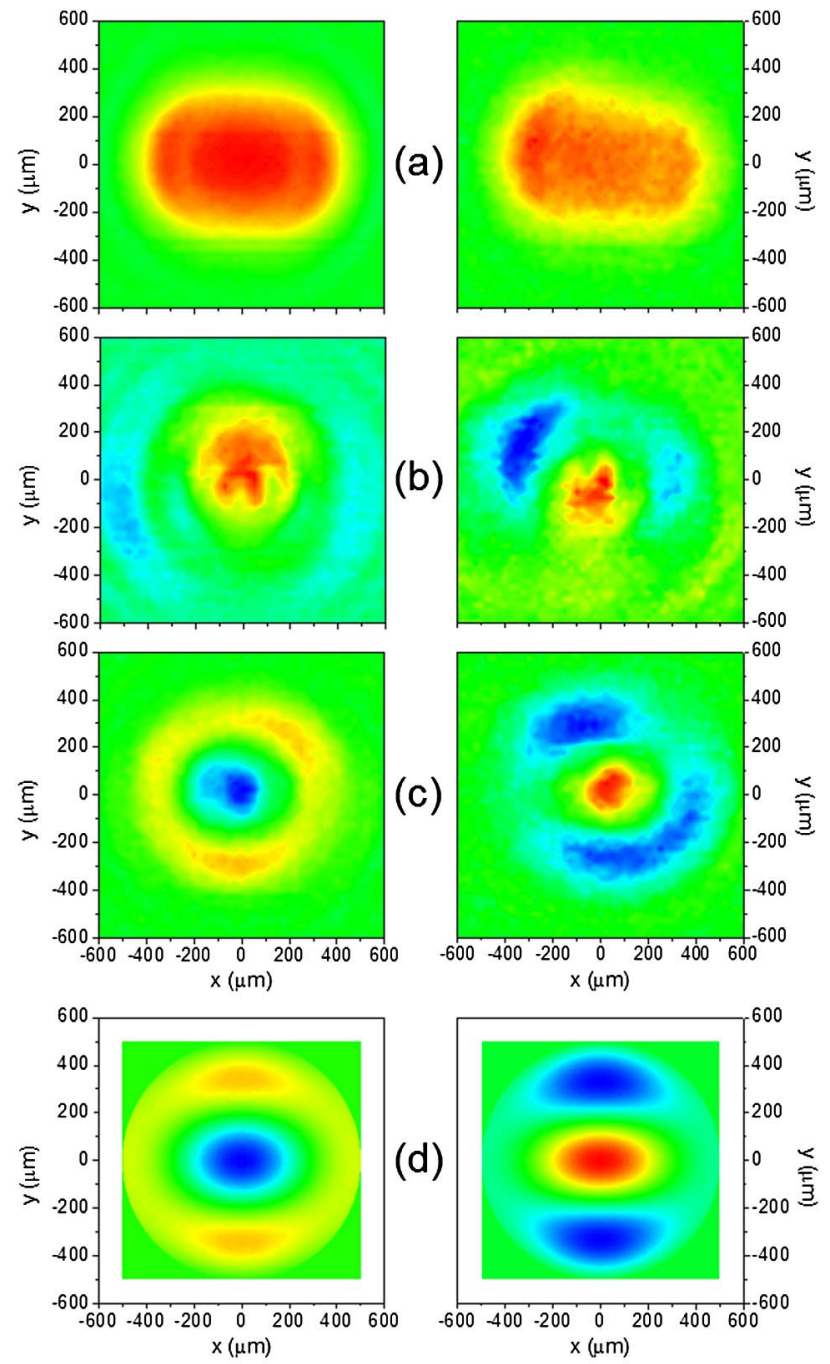

$E_{x}$

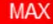

Fig. 4. (Color online) Experimental time-resolved normalized $E_{x}(x, y)$ profiles for the Ag-only (left panels) and AgI/Ag (right panels) waveguides for the time-delay: $\Delta t=1.5 \mathrm{ps}, 4.8 \mathrm{ps}$, and $7.5 \mathrm{ps}$ (a),(b), (c); and a mixture of numerically computed $\mathrm{TM}_{11}$ and $\mathrm{TE}_{12}$ modes at the frequency that makes them arrive at $\sim 7.5 \mathrm{ps}(\mathrm{d})$.

The fact that the field pattern at $7.5 \mathrm{ps}$ for the AgI-coated waveguide is different from the uncoated waveguide is partly caused by a higher attenuation experienced by the $\mathrm{TM}_{11}$ mode due to the dielectric coating. This suggestion is supported by the effect of the dielectric layer on the attenuation coefficients [Fig. 2(b)]. The $\mathrm{TM}_{11}$ mode contains field lines terminating on the guide walls, and thus a portion of the mode energy is travelling within the dielectric in the AgI-coated waveguide. Therefore, the dielectric coating increases the attenuation of the $\mathrm{TM}_{11}$ mode, whereas the $\mathrm{TE}_{12}$ mode, with the field approaching zero near the metallic surface, makes it less sensitive to the dielectric coating. Hence the field pattern in the AgI-coated waveguide is more similar to the $\mathrm{TE}_{12}$ mode.

The detailed characterization of the mode structure presented here provides a further insight into waveguide mode imaging using $\mathrm{CW} \mathrm{THz}$ sources or using the Fouriertransformed time-domain data. In the single frequency analysis, normal modes cannot be distinguished experimentally from multimode interference, similar to the example 
shown in Fig. 4(d). In the multimode operation, the output field (or intensity) pattern is therefore a superposition of all excited modes. Considering the normal modes determined in this study, we conclude that the $\mathrm{TE}_{11^{-}}, \mathrm{TM}_{11^{-}}$, and $\mathrm{TE}_{12^{-}}$like modes can be excited in the waveguide if the $\mathrm{THz}$ beam is centered on the waveguide axis. The output pattern therefore can take a form of any superposition of these modes.

As the first example, in Fig. 5(a) we show a combination of the fundamental modes at $3.2 \mathrm{THz}$ for the AgI-coated waveguide $\left(0.8930 \times E_{x}^{\mathrm{TE}_{11}}\right.$ and $\left.-0.45 \times E_{x}^{\mathrm{TM}_{11}}\right)$ that is similar to the pattern of the low-loss $\mathrm{HE}_{11}$ mode $[\underline{1}, \underline{15}, \underline{17}-19,30,31]$. If the $\mathrm{HE}_{11}$ mode pattern develops at the output aperture of the waveguide either from the fundamental $\mathrm{HE}_{11}$ mode or from the above combination, it is known to generate an intensity distribution with a Gaussian-like profile in the far field $[6,14,20]$. Notice that even at $3.2 \mathrm{THz}$, the frequency used in the $\mathrm{CW}$ experiment of [25], our numerical modeling shows the $\mathrm{TE}_{11^{-}}$and $\mathrm{TM}_{11^{-}}$like distributions because the dielectric coating is not thick enough to form the ideal low-loss $\mathrm{HE}_{11}$ mode.

To back up the above discussion, we perform a CW far-field mode imaging using a similar AgI-lined hollow metallic waveguide (140 mm long) [25]. Figure $\underline{6}$ shows that a Gaussian-like distribution is measured in the far field. It is important to note that this single $\mathrm{CW}$ experiment is not sufficient to determine if such a pattern has been originated from the $\mathrm{HE}_{11}$ mode or from a superposition of modes. Only after the near-field time-resolved analysis can we conclude that it is originated from the admixture $\mathrm{TE}_{11}+\mathrm{TM}_{11}$. This example shows that
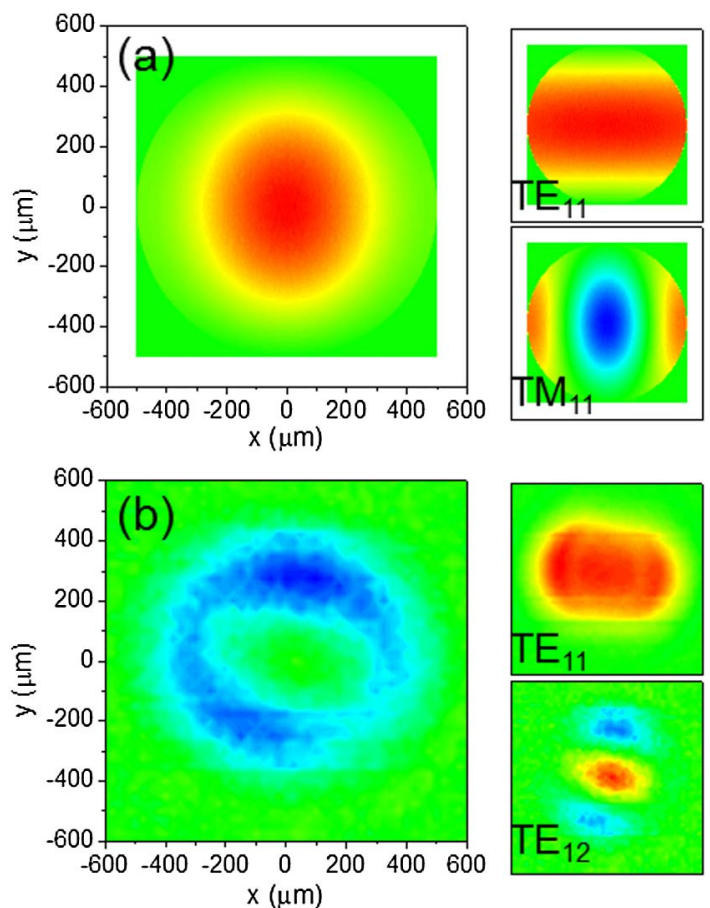

$$
\text { MIN } \quad E_{x}
$$

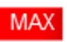

Fig. 5. (Color online) (a) Numerically computed $E_{x}(x, y)$ of the $\mathrm{TE}_{11}$ (top right) and $\mathrm{TM}_{11}$ (bottom right) at $3.2 \mathrm{THz}$ along with the resulting $E_{x}(x, y)$ when they are added out of phase with certain weights (left) for the AgI-lined cylindrical waveguide of [25]. (b) Experimental $E_{x}(x, y)$ profiles of the $\mathrm{TE}_{11}$ (top right) and $\mathrm{TE}_{12}$ (bottom right) modes in the AgI/Ag waveguide and their superposition (left).

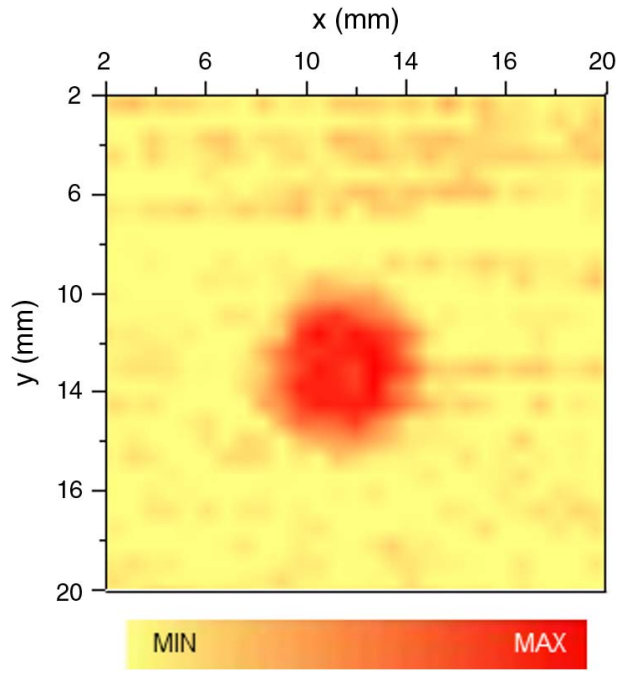

Fig. 6. (Color online) Far-field spatial intensity distribution of a $140 \mathrm{~mm}$ long waveguide with a $1.0 \mathrm{~mm}$ bore diameter having an ultrathin AgI coating of $1 \mu \mathrm{m}$ and excited by an azimuthally polarized microring distributed quantum cascade laser operating at $3.2 \mathrm{THz}$.

the impact of the dielectric coating on the hollow metallic waveguides is difficult to determine using a single frequency experiment.

As the second example we consider a superposition of two mode profiles experimentally determined in the time-resolved measurements: $-0.3 \times E_{x}^{\mathrm{TE}_{11}}$ and $0.9539 \times E_{x}^{\mathrm{TE}_{12}}$ [Fig. 5(b)]. The latter field pattern creates an intensity distribution similar to another important mode, the $\mathrm{TE}_{01}$ mode. As in the first example, CW experiment is not sufficient to determine if the pattern is caused by the $\mathrm{TE}_{01}$ mode or by a mode superposition. We note, however, that the latter ambiguity can be resolved using polarizers or polarization-sensitive detectors.

These examples demonstrate the importance of supplementing the mode images in multimode $\mathrm{THz}$ waveguides with the time-resolved waveguide mode imaging and numerical modeling. Applying similar analysis to the experimental results on AgI-coated waveguides in [25], in which the observed emission patterns suggested the presence of the $\mathrm{HE}_{11}$ mode, we conclude that the result may have been affected by mode interference.

In case the time-resolved characterization is not available, we can provide the following recommendations for waveguide mode characterization: (i) the length of the waveguide samples must be kept long enough so that only one or two modes maintain significant intensities; (ii) output patterns must be measured for several waveguide lengths to verify that the pattern remains independent of the length. In addition, $a$ priori knowledge of the normal modes and the corresponding losses is essential.

We can also make the following recommendations for timeresolved characterization: (i) control of the input beam can help in transferring most of the energy into a single mode, as it was done in [31] and here; and (ii) frequency-narrower $\mathrm{THz}$ probing pulses can reduce the temporal overlapping modes because with the frequency-narrower pulses experience a smaller temporal broadening. This can be done either by a $\mathrm{THz}$ source with narrower frequency bandwidth or by placing an appropriate filter between the broadband $\mathrm{THz}$ source and the waveguide. 


\section{CONCLUSIONS}

We characterized modes experimentally in Ag/AgI hollow metallic waveguides at $\mathrm{THz}$ frequencies, in the regime of ultrathin dielectric coating using the near-field imaging system combined with time-domain measurements. The experimental results are supplemented with electromagnetic simulations. For comparison purposes and for the sake of completeness, the identical methodology has been also applied to a wellknown cylindrical hollow metallic waveguide. We show that in the $\mathrm{THz}$ frequency range $(1-3.2 \mathrm{THz})$ the $1 \mu \mathrm{m}$ thick layer of AgI on the waveguide inner walls is not sufficient to form the ideal low-loss $\mathrm{HE}_{11}$ modes. The mode profiles for the lowestorder modes, as confirmed by numerical modeling, are more similar to the classical modes in the hollow metallic waveguide. We show here that, in order to bridge the gap between observations and understanding, it is extremely effective to combine $\mathrm{THz}$ near-field imaging, time-resolved measurements, and numerical electromagnetic modeling. With these complementary techniques, a complete picture of the problem can be captured, allowing one to identify unambiguously the modal structure in real $\mathrm{THz}$ waveguides in a multimode environment. The work is another step forward in the new but rapidly evolving subfield of $\mathrm{THz}$ near-field microscopy for waveguide characterization.

\section{ACKNOWLEDGMENTS}

The authors are grateful to Prof. M. Sorolla from Universidad Pública de Navarra for helpful comments. This work was supported by the Engineering and Physical Sciences Research Council (grant numbers EP/G033870/1 and EP/J017671/1) and the Royal Society (grant number UF080745). M.S.V. acknowledges financial support of the Italian Ministry of Education, University, and Research (MIUR) through the program FIRB-Futuro in Ricerca 2010 RBFR10LULP Fundamental research on Terahertz photonic devices.

\section{REFERENCES}

1. O. Mitrofanov, R. James, F. Aníbal Fernández, T. K. Mavrogordatos, and J. A. Harrington, "Reducing transmission losses in hollow THz waveguides," IEEE Trans. THz Sci. Tech. 1, 124-132 (2011).

2. E. A. J. Marcatilli and R. A. Schmeltzer, "Hollow metallic and dielectric waveguides for long distance optical transmission and lasers," Bell Syst. Tech. J. 43, 1783-1809 (1964).

3. J. W. Carlin and P. D'Agostino, "Normal modes in overmoded dielectric-lined circular waveguide," Bell Syst. Tech. J. 52, 453-486 (1973).

4. J. W. Carlin and A. Maione, "Experimental verification of lowloss TM modes in dielectric-lined waveguide," Bell Syst. Tech. J. 52, 487-496 (1973).

5. N. Marcuvitz, Waveguide Handbook, ser. Electromagnetic Waves Series (IEE, 1986).

6. P. J. B. Clarricoats and A. D. Olver, Corrugated Horns for Microwave Antennas (IEE, 1984).

7. P. Bhartia and I. J. Bahl, Millimeter Wave Engineering and Applications (Wiley, 1984).

8. J. P. Crenn, "A study of waveguides for far infrared interferometers measuring electron density of Tokamak plasmas," IEEE Trans. Microwave Theory Tech. 27, 573-577 (1979).

9. J. L. Doane, "Propagation and mode coupling in corrugated and smooth-wall circular waveguides," in Infrared and Millimeter Waves, K. J. Button, ed., vol. 13 (Academic, 1985), pp. 123-170.

10. M. Miyagi, K. Harada, and S. Kawakami, "Wave propagation and attenuation in the general class of circular hollow waveguides with uniform curvature," IEEE Trans. Microwave Theory Tech. 32, 513-521 (1984).
11. B. Bowden, J. A. Harrington, and O. Mitrofanov, "Low-loss modes in hollow metallic terahertz waveguides with dielectric coatings," Appl. Phys. Lett. 93, 181104 (2008).

12. M. Thumm, A. Jacobs, and M. Sorolla Ayza, "Design of short high-power $\mathrm{TE}_{11}-\mathrm{HE}_{11}$ mode converters in highly overmoded corrugated waveguides," IEEE Trans. Microwave Theory Tech. 39, 301-309 (1991).

13. B. Z. Katsenelenbaum, L. Mercader del Rio, M. Pereyaslavets, M. Sorolla Ayza, and M. Thumm, Theory of Nonuniform Waveguides: The Cross-Section Method (Institute of Electrical Engineers, 1998).

14. J. Teniente, R. Gonzalo, C. Del Río, J. Martí Canales, M. Sorolla A. Fernández, K. Likin, and R. Martín, "Corrugated horn antenna for low-power testing of the quasioptical transmission lines at TJ-II Stellerator," J. Infrared Millimeter Waves 20, 1-19 (1999).

15. M. Miyagi and S. Kawakami, "Design theory of dielectric-coated circular metallic waveguides for infrared transmission," J. Lightwave Technol. 2, 116-126 (1984).

16. J. A. Harrington, Infrared Fiber Optics and Their Applications (SPIE, 2004).

17. Y. Kato and M. Miyagi, "Modes and attenuation constants in circular hollow waveguides with small core diameters for the infrared," IEEE Trans. Microwave Theory Tech. 40, 679-685 (1992).

18. Y. Kato and M. Miyagi, "Numerical analysis of mode structures and attenuations in dielectric-coated circular hollow waveguides for the infrared," IEEE Trans. Microwave Theory Tech. 42, 2336-2342 (1994).

19. B. Bowden, J. A. Harrington, and O. Mitrofanov, "Silver/ polystyrene-coated hollow glass waveguides for the transmission of terahertz radiation,” Opt. Lett. 32, 2945-2947 (2007).

20. G. Gallot, S. P. Jamison, R. W. McGowan, and D. Grischkowsky, “Terahertz waveguides," J. Opt. Soc. Am. B 17, 851-863 (2000).

21. E. de Rijk, A. Macor, J.-P. Hogge, S. Alberti, and J.-P. Ansermet, "Note: stacked rings for terahertz wave-guiding," Rev. Sci. Instrum. 82, 066102 (2011)

22. M. Alaluf, J. Dror, R. Dahan, and N. Croitoru, "Plastic hollow fibers as a selective infrared radiation transmitting medium," J. Appl. Phys. 72, 3878-3883 (1992).

23. K. Iwai, A. Hongo, H. Takaku, M. Miyagi, J.-i. Ishiyama, X.-X. Wu, Y.-W. Shi, and Y. Matsuura, "Fabrication and transmission characteristics of infrared hollow fiber based on silver-clad stainless steel pipes," Appl. Opt. 48, 6207-6212 (2009).

24. X.-L. Tang, Y.-W. Shi, Y. Matsuura, K. Iwai, and M. Miyagi, "Transmission characteristics of terahertz hollow fiber with an absorptive dielectric inner-coating film," Opt. Lett. 34, 2231-2233 (2009).

25. U. S. de Cumis, J.-H. Xu, C. M. Bledt, J. A. Harrington, A Tredicucci, and M. S. Vitiello, "Flexible, low-loss waveguide designs for efficient coupling to quantum cascade lasers in the far-infrared," J. Infrared Milli. THz Waves 33, 319-326 (2012).

26. M. S. Vitiello, J.-H. Xu, F. Beltram, A. Tredicucci, O. Mitrofanov, J. A. Harrington, H. E. Beere, and D. A. Ritchie, "Guiding a terahertz quantum cascade laser into a flexible silver-coated waveguide," J. Appl. Phys. 110, 063112 (2011).

27. M. S. Vitiello, J.-H. Xu, M. Kumar, F. Beltram, A. Tredicucci, O. Mitrofanov, H. E. Beere, and D. A. Ritchie, "High efficiency coupling of terahertz micro-ring quantum cascade lasers to the low-loss optical modes of hollow metallic waveguides," Opt. Express 19, 1122-1130 (2011).

28. O. Mitrofanov, I. Brener, R. Harel, J. D. Wynn, L. N. Pfeiffer, K. W. West, and J. Federici, "Terahertz near-field microscopy based on a collection mode detector," Appl. Phys. Lett. 77, 3496-3498 (2000).

29. O. Mitrofanov, M. Lee, J. W. P. Hsu, I. Brener, R. Harel, J. Federici, J. D. Wynn, L. N. Pfeiffer, and K. W. West, "Collection-mode near-field imaging with $0.5 \mathrm{THz}$ pulses," IEEE J. Sel. Top. Quantum Electron. 7, 600-607 (2001).

30. O. Mitrofanov, T. Tan, P. R. Mark, B. Bowden, and J. A. Harrington, "Waveguide mode imaging and dispersion analysis with terahertz near-field microscopy," Appl. Phys. Lett. 94, 171104 (2009).

31. O. Mitrofanov and J. A. Harrington, "Dielectric-lined cylindrical metallic THz waveguides: mode structure and dispersion," Opt Express 18, 1898-1903 (2010). 
32. C. M. Bledt, J. A. Harrington, and J. M. Kriesel, "Loss and modal properties of Ag/AgI hollow glass waveguides," Appl. Opt. 51, 3114-3119 (2012).

33. C. Dragone, "Attenuation and radiation characteristics of the $\mathrm{HE}_{11}$ mode," IEEE Trans. Microwave Theory Tech. 28, 704-710 (1980).
34. C. S. Lee, S. W. Lee, and S. L. Chuang, "Plot of modal field distribution in rectangular and circular waveguides," IEEE Trans. Microwave Theory Tech. 33, 271-274 (1985).

35. Y. Matsuura and E. Takeda, "Hollow optical fibers loaded with an inner dielectric film for terahertz broadband spectroscopy," J. Opt. Soc. Am. B 25, 1949-1954 (2008). 